\title{
PRELIMINARY TEST ESTIMATION AND SHRINKAGE PRELIMINARY TEST ESTIMATION IN NORMAL AND NEGATIVE EXPONENTIAL DISTRIBUTION USING LINEX LOSS FUNCTION
}

\author{
Dr. Binod Kumar Singh \\ University of Petroleum \& Energy Studies, Dehradun, India
}

\begin{abstract}
In statistical estimation procedure prior information regarding the unknown value of parameter is utilizing and it may result in a decrease of sampling variability of the estimator or it may save sample size which is desirable in many estimation procedures. The commonly used approaches in statistical inference which utilize prior information are Bayesian approach, preliminary test procedure and shrinkage estimation. The paper proposes preliminary test estimator and shrinkage preliminary test estimator for the variance in normal distribution and studies its property under Linex loss function. The paper also proposes and suggests shrinkage preliminary test estimator for the variance in negative exponential distribution and studies its property under Linex loss function.
\end{abstract}

\section{Key words}

Linex loss function, Shrinkage estimation, Preliminary test estimation, Shrinkage preliminary test estimation

\section{INTRODUCTION}

Thompson (1968) introduces the idea of shrinkage estimation and found that the shrinkage estimator perform better if the guess value is in the vicinity of true value and when sample size is small. In many practical problems it may not be known whether a prior value $\left(\theta_{0}\right)$ is close to the true value of the parameter [3]. It $H_{0}: \theta=\theta_{0}$ is accepted, thenthe shrinkage estimator otherwise the usual estimator can be used.[2],[4-8], [10-11], [13], [15-21], [24], [26-28] and [31] have used preliminary test estimator and shrinkage estimator in different distributions. [25] showed that the non-optimality of preliminary test estimator for mean in normal, binomial and Poisson distribution.[14] proposed shrinkageestimator for the mean in an exponential distribution under type II censoring data. [2] extended the above estimator tomean ( $\theta$ )in an exponential distribution by acceptance region of uniformly most powerful test with a level of significance (for testing the hypothesis [12] used a different weight $\mathrm{R}$ which is more conservative than the above in the sense that test statistics is near to the boundary of the critical region if $k=1$. This suggests that the use of the test statistic for preliminary test estimator in the construction of weight function $\mathrm{k}$ is more 
reasonable than fixed or pre-determined value of k. [9]proposed modified double stage shrinkage estimator.

In the context of real estate assessment, [29] proposed an asymmetric loss function called Linex loss function (linear-exponential) as

$$
L(a, \Delta)=e^{a \Delta}-c \Delta-1,-\infty<\Delta<\infty
$$

Where a, $\mathrm{c}>0$.

The Linex loss function is employed in the analysis of several central statistical estimation and prediction problems. The Linex loss function which rises exponentially on one side of zero and almost linearly on the other side of zero. This loss function behaves linearly for large underestimation errors $(\Delta<0)$, in which case the exponential term vanishes and exponentially for large over-estimation errors $(\Delta>0)$, in which case the exponential term dominates and vanishes when there is no estimation $(\Delta=0)$.

[31] points out that if $\mathrm{c}=\mathrm{a}$ then equation (1.1) is minimized at $\Delta=0$.With this restriction equation (1.1) reduces to

$$
L(a, \Delta)=e^{a \Delta}-a \Delta-1,-\infty<\Delta<\infty
$$

[31] points out that for negative values of a Linex loss retains its linear-exponential character, though for opposite estimation error, and that for small values of $|a|$ Linex loss is nearly symmetric and approximately proportional to squared error loss. But for larger value of $|a|$ it is quite asymmetric.

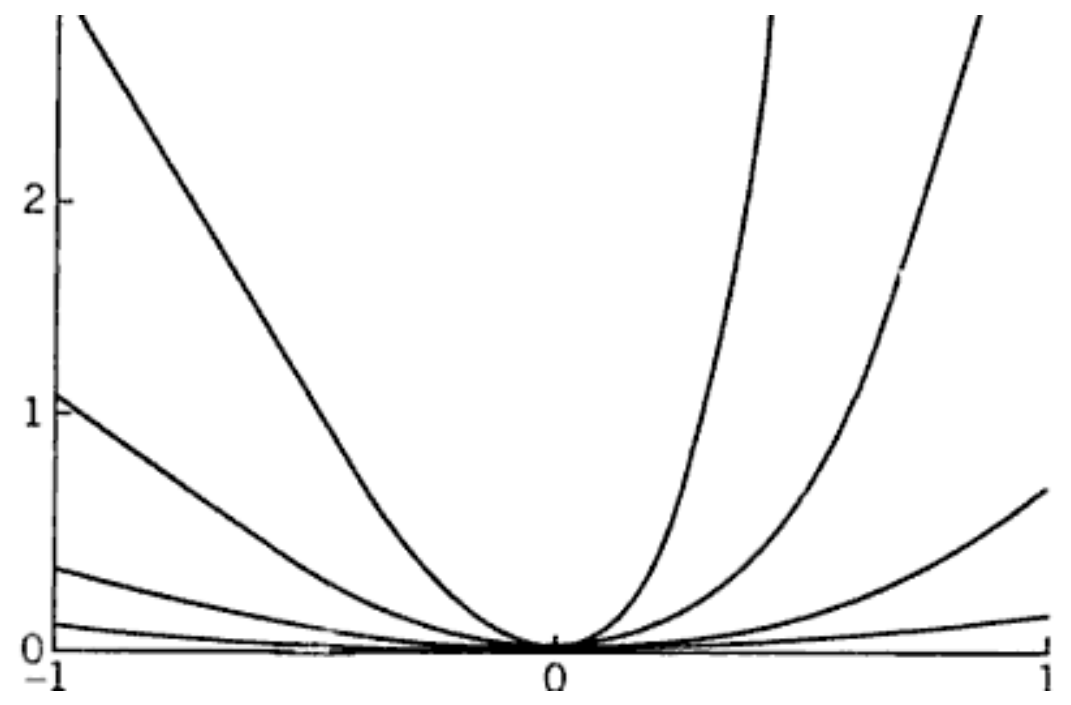

Linex loss function 
An example is given in the field of hydrology with the estimation of peak water level in the construction of the dam. In that case, overestimation represents a conservative error which increases construction costs, while underestimation corresponds to the much more serious error in which overflows might lead to huge damage in the adjacent area.

The Linex loss function (another form) is

$$
L\left(a, \Delta^{*}\right)=b\left(e^{a \Delta^{*}}-c \Delta^{*}-1\right), \quad \Delta^{*}=\frac{\Lambda}{\mu}-1, \quad a \neq 0
$$

Where $\mathrm{a}$ and $\mathrm{b}$ are shaped and scale parameter. If Linex loss reduced to square error.

In section 2, proposed preliminary test estimator for variance in normal distribution as

$$
\hat{\sigma}_{P T}^{2}=\left\{\begin{array}{cc}
\sigma_{0}^{2} & \text { if } \mathrm{H}_{0}: \sigma^{2}=\sigma_{0}^{2} \text { is accepted } \\
\frac{\mathrm{n}-1}{2 \mathrm{a}}\left(1-\mathrm{e}^{-\frac{2 \mathrm{a}}{\mathrm{n}+1}}\right) \mathrm{s}^{2}=\mathrm{P} & \text { otherwise }
\end{array}\right.
$$

Andstudies its properties under Linex loss function.

Here $\frac{n-1}{2 a}\left(1-\mathrm{e}^{-\frac{2 a}{n+1}}\right) s^{2}$ is the improve estimator in the class of estimatorY $=\operatorname{cs}^{2}$ under the Linex loss function [23].

In section 3, proposed shrinkagepreliminary test estimator for $\sigma^{2}$ in normal distribution and studied its property under Linex loss function and also suggests another shrinkage preliminary test estimator for $\sigma^{2}$ in normal distribution.

The proposed shrinkage preliminary test estimator for $\sigma^{2}$ in normal distribution as

$$
\left.\sigma_{S P T_{1}}^{2}=\left[\begin{array}{l}
\left.\frac{(\mathrm{n}-1) s^{2}}{\left(\ell_{2}-\ell_{1}\right) \sigma_{0}^{2}}-\frac{\ell_{1}}{\ell_{2}-\ell_{1}}\right) s^{2}+\left\{1-\frac{(\mathrm{n}-1) s^{2}}{\left(\ell_{2}-\ell_{1}\right) \sigma_{0}^{2}}+\frac{\ell_{1}}{\ell_{2}-\ell_{1}}\right\} \sigma_{0}^{2} \text { if } \ell_{1} \leq \frac{(\mathrm{n}-1) s^{2}}{\sigma_{0}^{2}} \leq \ell_{2} \\
\frac{n-1}{2 a}\left(1-\mathrm{e}^{-\frac{2 a}{n+1}}\right) s^{2}=\mathrm{P}, \text { otherwise }
\end{array}\right] 1.5\right)
$$

The other proposesshrinkage preliminary test estimatorby taking $k^{2}=k_{1}$ may be defined as 
International Journal of Soft Computing, Mathematics and Control (IJSCMC), Vol.4, No. 1,February 2015

$$
\hat{\sigma}_{S P T_{2}}^{2}=\left\{\begin{array}{cc}
k_{1} s^{2}+\left(1-k_{1}\right) \sigma_{0}^{2} & \text { if } \ell_{1} \leq \frac{(\mathrm{n}-1) s^{2}}{\sigma_{0}^{2}} \leq \ell_{2} \\
\frac{n-1}{2 a}\left(1-\mathrm{e}^{-\frac{2 a}{n+1}}\right) s^{2}=P & \text { otherwise }
\end{array}\right.
$$

In section 4, proposedshrinkage preliminary test estimatorofvariance $\left(\theta^{2}\right)$ in negative exponential distribution and studied its property under Linex loss function and also suggest another shrinkage preliminary test estimator for the variance $\left(\theta^{2}\right)$ in negative exponential distribution.

$$
\hat{\theta}_{S P T_{1}}^{2}=\left[\begin{array}{l}
\left(\frac{2 \mathrm{n} \overline{\mathrm{x}}}{\left(\ell_{2}-\ell_{1}\right) \theta_{0}}-\frac{\ell_{1}}{\ell_{2}-\ell_{1}}\right) \bar{x}^{2}+\left\{1-\frac{2 \mathrm{n} \overline{\mathrm{x}}}{\left(\ell_{2}-\ell_{1}\right) \theta_{0}}+\frac{\ell_{1}}{\ell_{2}-\ell_{1}}\right\} \theta_{0}^{2} \quad \text { if } \frac{\ell_{1} \theta_{0}}{2 n} \leq \bar{x} \leq \frac{\ell_{2} \theta_{0}}{2 n} \\
\frac{n^{2}}{(n+2)(n+3)}\left(1-\frac{\mathrm{c}}{\mathrm{n}}\right) \overline{\mathrm{x}}^{2}=\mathrm{Y}, \text { otherwise }
\end{array}\right]
$$

The other proposes shrinkage preliminary test estimator by taking $k^{2}=k_{1}$ may be defined as

$$
\hat{\theta}_{S P T_{2}}^{2}=\left[\begin{array}{l}
\left(\frac{2 \mathrm{n} \overline{\mathrm{x}}}{\left(\ell_{2}-\ell_{1}\right) \theta_{0}}-\frac{\ell_{1}}{\ell_{2}-\ell_{1}}\right)^{2} \bar{x}^{2}+\left\{1-\left(\frac{2 \mathrm{n} \overline{\mathrm{x}}}{\left(\ell_{2}-\ell_{1}\right) \theta_{0}}+\frac{\ell_{1}}{\ell_{2}-\ell_{1}}\right)^{2}\right\}^{2} \theta_{0}^{2} \text { if } \frac{\ell_{1} \theta_{0}}{2 n} \leq \bar{x} \leq \frac{\ell_{2} \theta_{0}}{2 n} \\
\frac{n^{2}}{(n+2)(n+3)}\left(1-\frac{\mathrm{c}}{\mathrm{n}}\right) \overline{\mathrm{x}}^{2}=\mathrm{Y} \quad \text { otherwise }
\end{array}\right]
$$

The value of $\mathrm{c}$ <and, since the magnitude of the shrinkage factor under Linex loss is smaller than the mean square criterion.

\section{PRELIMINARY TEST ESTIMATOR FOR VARIANCE IN NORMAL DISTRIBUTION UNDER LINEX LOSS FUNCTION}

Let us consider a normal distribution with $N\left(\mu, \sigma^{2}\right)$ and also let $\sigma_{0}^{2}$ is the prior value of $\sigma^{2}$, thepreliminary test estimator is

$$
\hat{\sigma}_{P T}^{2}=\left[\begin{array}{l}
\sigma_{0}^{2} \text { if } \ell_{1} \leq \frac{(\mathrm{n}-1) s^{2}}{\sigma_{0}^{2}} \leq \ell_{2} \\
\frac{n-1}{2 a}\left(1-\mathrm{e}^{\left.-\frac{2 a}{n+1}\right) \mathrm{s}^{2}}=P,\right. \text { otherwise }
\end{array}\right]
$$


International Journal of Soft Computing, Mathematics and Control (IJSCMC), Vol.4, No. 1,February 2015

Here, $P\left[\chi_{n-1}^{2} \leq \ell_{1}\right]=P\left[\chi_{n-1}^{2} \geq \ell_{2}\right]=\alpha / 2$.

The improve estimator of $\sigma^{2}$ under the Linex loss function is

$\frac{n-1}{2 a}\left(1-\mathrm{e}^{-\frac{2 a}{n+1}}\right) \mathrm{s}^{2}=\left[\frac{\mathrm{n}-1}{n+1}-\frac{\mathrm{a}(\mathrm{n}-1)}{(\mathrm{n}+1)^{2}}+\cdots\right] \mathrm{s}^{2}=\mathrm{P}[23]$

$\hat{\sigma}_{P T}^{2}$ under mean square error was considered previously. The risk under an invariant form ofLinex lossfunction is defined as

$$
\begin{aligned}
R\left(a, \Delta^{*}\right) & =\mathrm{E}\left[\mathrm{L}\left(a, \Delta^{*}\right)\right]=\mathrm{E}\left\{\mathrm{e}^{a\left(\frac{\sigma_{0}^{2}}{\sigma^{2}}-1\right)}-\mathrm{a}\left(\frac{\sigma_{0}^{2}}{\sigma^{2}}-1\right)-1\right\}, \operatorname{Pr}\left\{\frac{\ell_{1} \sigma_{0}^{2}}{n-1} \leq \mathrm{s}^{2} \leq \frac{\ell_{2} \sigma_{0}^{2}}{n-1}\right\} \\
& +\mathrm{E}\left\{\mathrm{e}^{a\left(\frac{P}{\sigma^{2}}-1\right)}-\mathrm{a}\left(\frac{P}{\sigma^{2}}-1\right)-1\right\}, \operatorname{Pr}\left\{s^{2} \leq \frac{\ell_{1} \sigma_{0}^{2}}{n-1} \text { or } \mathrm{s}^{2} \geq \frac{\ell_{2} \sigma_{0}^{2}}{(n-1)}\right\}
\end{aligned}
$$

Putting $\frac{(\mathrm{n}-1) s^{2}}{2 \sigma^{2}}=\mathrm{t}, s^{2}=\frac{2 \sigma^{2}}{\mathrm{n}-1} \mathrm{t}, d s^{2}=\left(\frac{2 \sigma^{2}}{\mathrm{n}-1}\right) \mathrm{dt}$. Thus,

$\frac{2}{a^{2}} R\left(a, \Delta^{*}\right)=\left[\left(\frac{\sigma_{0}^{2}}{\sigma^{2}}-1\right)^{2}+\frac{\mathrm{a}}{3}\left(\frac{\sigma_{0}^{2}}{\sigma^{2}}-1\right)^{3}+\frac{\mathrm{a}^{2}}{4.3}\left(\frac{\sigma_{0}^{2}}{\sigma^{2}}-1\right)^{4}+\cdots\right] \int_{\frac{\ell_{1} \sigma_{0}^{2}}{2 \sigma^{2}(n-1)}}^{\frac{\ell_{2} \sigma_{0}^{2}}{2 \sigma^{2}(n-1)}} \frac{1}{\operatorname{Gamma}\left[\frac{n-1}{2}\right]} \mathrm{e}^{-t} \mathrm{t}^{\frac{n-1}{2}-1} d t$

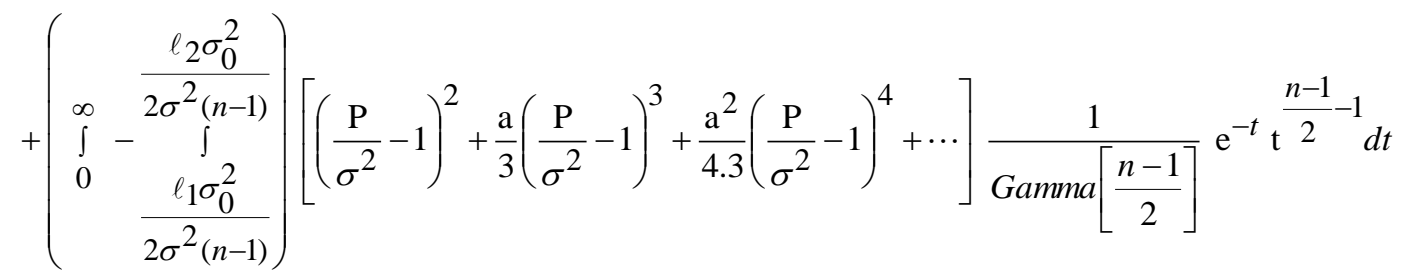

Let $\frac{\sigma_{0}^{2}}{\sigma^{2}}=\delta, \quad \frac{P}{\sigma^{2}}=\left\{\frac{\mathrm{n}-1}{n+1}-\frac{\mathrm{a}(\mathrm{n}-1)}{(n+1)^{2}}+\cdots\right\} \frac{2}{(n-1)} \mathrm{t}=\left\{\frac{2}{\mathrm{n}+1}-\frac{2 \mathrm{a}}{(\mathrm{n}+1)^{2}}+\cdots\right\} \mathrm{t}$,

Thus, 
International Journal of Soft Computing, Mathematics and Control (IJSCMC), Vol.4, No. 1,February 2015

$$
\begin{aligned}
& \frac{2}{a^{2}} R\left(a, \Delta^{*}\right)=\left[(\delta-1)^{2}+\frac{\mathrm{a}}{3}(\delta-1)^{3}+\frac{\mathrm{a}^{2}}{4.3}(\delta-1)^{4}+\cdots\right]_{\frac{\ell_{1} \delta}{2(n-1)}}^{\frac{\ell_{2} \delta}{2(n-1)}} \frac{1}{\sqrt{\mathrm{n}-1}} \mathrm{e}^{-t} \mathrm{t}^{\frac{n-1}{2}-1} d t+\left(\int_{0}^{\infty}-\int_{\frac{\ell_{1} \delta}{2(n-1)}}^{\frac{\ell_{2} \delta}{2(n-1)}}\right) \\
& {\left[\left\{-\left(\frac{n-1}{n+1}\right)-\frac{2 \mathrm{a}}{(\mathrm{n}+1)^{2}}+\cdots\right\}^{2}+\frac{\mathrm{a}}{3}\left\{-\frac{\mathrm{n}-1}{n+1}-\frac{2 \mathrm{a}}{(\mathrm{n}+1)^{2}}+\cdots\right\}^{3}+\frac{\mathrm{a}^{2}}{4.3}\{\}^{4}+\cdots\right] \frac{\mathrm{e}^{-t} \mathrm{t}^{\frac{n-1}{2}-1}}{\Gamma\left(\frac{n-1}{2}\right)} d t}
\end{aligned}
$$

If $|a| \rightarrow 0$, thus

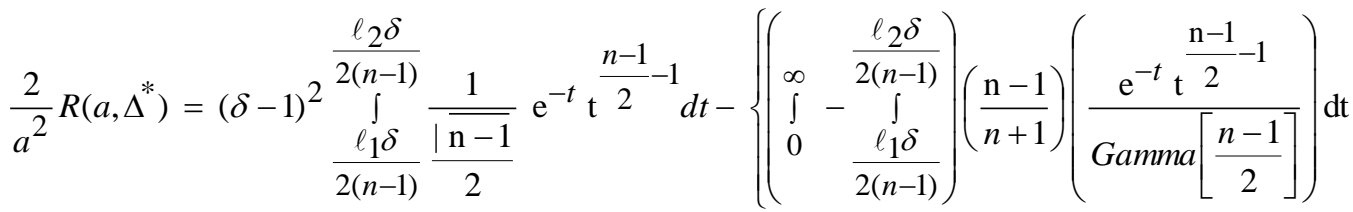

$$
\begin{aligned}
& =(\delta-1)^{2} \underset{\frac{\ell_{1} \delta}{2(n-1)}}{\int_{2} \frac{\ell_{2} \delta}{2(n-1)}} \frac{1}{\text { Gamma }\left[\frac{n-1}{2}\right]} \mathrm{e}^{-t} \mathrm{t}^{\frac{n-1}{2}-1} d t-\left\{\left(\left(\begin{array}{cc}
\infty & \frac{\ell_{2} \delta}{2(n-1)} \\
\int_{0} & -\int_{1} \delta \\
2(n-1)
\end{array}\right)\left(\frac{\mathrm{n}-1}{n+1}\right)\left(\frac{\mathrm{e}^{-t} \mathrm{t}^{\frac{\mathrm{n}-1}{2}-1}}{\text { Gamma }\left[\frac{n-1}{2}\right]}\right) \mathrm{dt}\right.\right. \\
& \text { If } \frac{\sigma_{0}^{2}}{\sigma^{2}}=1 \Rightarrow \delta=1 \text { and } \int_{\frac{\ell_{1} \delta}{2(n-1)}}^{\frac{\ell_{2} \delta}{2(n-1)}} \frac{1}{\operatorname{Gamma}\left[\frac{n-1}{2}\right]} \mathrm{e}^{-t} \mathrm{t}^{\frac{n-1}{2}-1} d t=1 \Rightarrow \ell_{1}=0, \ell_{2}=\infty
\end{aligned}
$$

Then, $\frac{2}{a^{2}} R\left(a, \Delta^{*}\right)=0$

The relative efficiency of estimator $\hat{\sigma}_{P T}^{2}$ with respect to Pis calculated for $\left(\sigma_{0}{ }^{2} / \sigma^{2}\right)=\delta=0.6(.2)$ (1.2), $\alpha=5 \%, a=.2$ (. 2)1.0 and $n=5$ (5)15 in table from 2.1 to 2.4. The tables show that the preliminary test estimator $\hat{\sigma}_{P T}^{2}$ performs better if, $0.2 \leq a \leq 1$ and $\mathrm{n}$ is less than 20.The maximum result is at the point $\delta=1$.

\section{SHRINKAGEPRELIMINARY FORVARIATION IN A NORMAL DISTRIBUTION UNDER LINEX LOSS FUNCTION}

[27] and[28] introduced shrunken estimator in life testing distribution. [21] introduced preliminary test estimator for the variance in normal distribution as 
International Journal of Soft Computing, Mathematics and Control (IJSCMC), Vol.4, No. 1,February 2015

$\hat{\sigma}^{2}=\left[\begin{array}{ll}k s^{2}+(1-k) \sigma_{0}^{2} & \text { if } \mathrm{H}_{0}: \sigma^{2}=\sigma_{0}^{2} \text { is accepted } \\ \mathrm{s}^{2}, \text { otherwise }\end{array}\right]$

Let us consider the class of estimator $Y=W s^{2}$ and find the value of W for which $\operatorname{MSE}(\mathrm{Y})$ is minimized, thus $W=\frac{n-1}{n+1}$ and the improve estimator is $Y=\frac{(n-1) s^{2}}{n+1}$ with $\operatorname{MSE}(Y)=\frac{2 \sigma^{4}}{n+1}$.

[31]considered Linex loss function, which performs better under the class of estimator $Y^{\prime}=W^{\prime} s^{2}$ where $W^{\prime}=\frac{\mathrm{n}-1}{2 a}\left(1-\mathrm{e}^{-\frac{2 a}{n+1}}\right)$ where $\mathrm{a} \neq 0$.

Let us consider shrinkagepreliminary test estimator for the variance in normal distribution as

$$
\hat{\sigma}_{S P T}^{2}=\left\{\begin{array}{cc}
\mathrm{ks}^{2}+(1-\mathrm{k}) \sigma_{0}^{2} & \text { if } \ell_{1} \leq \frac{(\mathrm{n}-1) \mathrm{s}^{2}}{\sigma_{0}^{2}} \leq \ell_{2} \\
\frac{\mathrm{n}-1}{2 \mathrm{a}}\left(1-\mathrm{e}^{-\frac{2 \mathrm{a}}{\mathrm{n}+1}}\right) \mathrm{s}^{2}=\mathrm{P} & \text { otherwise }
\end{array}\right.
$$

Here $\quad P\left[\chi_{n-1}^{2} \leq \ell_{1}\right]=\mathrm{P}\left[\chi_{n-1}^{2} \geq \ell_{2}\right]=\alpha / 2$.

Thus $\ell_{1} \leq \frac{(\mathrm{n}-1) s^{2}}{\sigma_{0}^{2}} \leq \ell_{2} \Rightarrow \theta \leq \frac{(\mathrm{n}-1) s^{2}}{\left(\ell_{2}-\ell_{1}\right) \sigma_{0}^{2}}-\frac{\ell_{1}}{\left(\ell_{2}-\ell_{1}\right)} \leq 1$

Let us suppose that shrunken factor $k=\frac{(\mathrm{n}-1) s^{2}}{\left(\ell_{2}-\ell_{1}\right) \sigma_{0}^{2}}-\frac{\ell_{1}}{\left(\ell_{2}-\ell_{1}\right)}$

And $k_{1}=\left\{\frac{(\mathrm{n}-1) s^{2}}{\left(\ell_{2}-\ell_{1}\right) \sigma_{0}^{2}}-\frac{\ell_{1}}{\ell_{2}-\ell_{1}}\right\}^{2}$

[12] suggested that the value of k may be taken as the function of test statistics.

Since, $\frac{(n-1) s^{2}}{\sigma_{0}^{2}}$ is the test criterion for testing of hypothesis $H_{0}: \sigma^{2}=\sigma_{0}^{2}$ against $H_{1}: \sigma^{2}=\sigma_{0}^{2}$. The acceptance region has received from 
International Journal of Soft Computing, Mathematics and Control (IJSCMC), Vol.4, No. 1,February 2015

$\operatorname{Pr}\left[\ell_{1} \leq \frac{(n-1) s^{2}}{\sigma_{0}^{2}} \leq \ell_{2}\right]=1-\alpha \Rightarrow \operatorname{Pr}\left[\frac{\ell_{1} \sigma_{0}^{2}}{n-1} \leq s^{2} \leq \frac{\ell_{2} \sigma_{0}^{2}}{n-1}\right]=1-\alpha$.

Thus the proposed shrunken preliminary test estimators are

$\hat{\sigma}_{S P T_{1}}^{2}=\left[\begin{array}{ll}\left\{\frac{(\mathrm{n}-1) s^{2}}{\sigma_{0}^{2}\left(\ell_{2}-\ell_{1}\right)}-\frac{\ell_{1}}{\ell_{2}-\ell_{1}}\right\} s^{2}+\left\{-\frac{(\mathrm{n}-1) s^{2}}{\sigma_{0}^{2}\left(\ell_{2}-\ell_{1}\right)}+\left(1+\frac{\ell_{1}}{\ell_{2}-\ell_{1}}\right)\right\} \sigma_{0}^{2} & \text { if } \ell_{1} \leq \frac{(\mathrm{n}-1) s^{2}}{\sigma_{0}^{2}} \leq \ell_{2} \\ \frac{n-1}{2 a}\left(1-\mathrm{e}^{-\frac{2 a}{n+1}}\right) \mathrm{s}^{2}=\mathrm{P}, \text { otherwise } & \end{array}\right]$

And

$\hat{\sigma}_{S P T_{2}}^{2}=\left[\begin{array}{ll}\left\{\frac{(\mathrm{n}-1) s^{2}}{\sigma_{0}^{2}\left(\ell_{2}-\ell_{1}\right)}-\frac{\ell_{1}}{\ell_{2}-\ell_{1}}\right\}^{2} s^{2}+\left\{1-\frac{(\mathrm{n}-1) s^{2}}{\sigma_{0}^{2}\left(\ell_{2}-\ell_{1}\right)}-\frac{\ell_{1}}{\ell_{2}-\ell_{1}}\right\}^{2} \sigma_{0}^{2} & \text { if } \ell_{1} \leq \frac{(\mathrm{n}-1) s^{2}}{\sigma_{0}^{2}} \leq \ell_{2} \\ \frac{n-1}{2 a}\left(1-\mathrm{e}^{-\frac{2 a}{n+1}}\right) \mathrm{s}^{2}=\mathrm{P} \text {, otherwise } & \end{array}\right]$

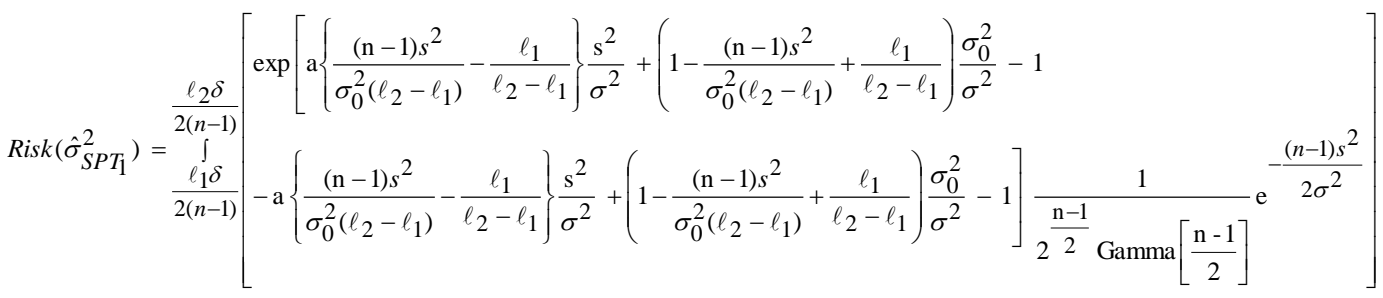

$+\left[\left\{\int_{0}^{\infty}-\frac{\ell_{2} \sigma_{0}^{2}}{(n-1)}\right\} \exp \left\{\mathrm{a}\left(\frac{\mathrm{n}-1}{2 a}\right)\left(1-\mathrm{e}^{-\frac{2 a}{n+1}}\right) \frac{s^{2}}{\sigma^{2}}\right\}-\mathrm{a}\left\{\left(\frac{\mathrm{n}-1}{2 a}\right)\left(1-\mathrm{e}^{-\frac{2 a}{n+1}}\right) \frac{s^{2}}{\sigma^{2}}-1\right\}-1\right]$

$\left.-\mathrm{a}\left\{\frac{(\mathrm{n}-1) s^{2}}{\sigma_{0}^{2}\left(\ell_{2}-\ell_{1}\right)}-\frac{\ell_{1}}{\ell_{2}-\ell_{1}}\right\} \frac{\mathrm{s}^{2}}{\sigma^{2}}+\left(1-\frac{(\mathrm{n}-1) s^{2}}{\sigma_{0}^{2}\left(\ell_{2}-\ell_{1}\right)}+\frac{\ell_{1}}{\ell_{2}-\ell_{1}}\right) \frac{\sigma_{0}^{2}}{\sigma^{2}}-1\right]$

$\frac{1}{2 \frac{\mathrm{n}-1}{2} \frac{\sqrt{n-1}}{2}} \mathrm{e}^{-\frac{(n-1) s^{2}}{2 \sigma^{2}}\left(\frac{(n-1) s^{2}}{\sigma^{2}}\right)^{\frac{n-1}{2}-1}\left(\frac{n-1}{\sigma^{2}}\right) d s^{2}}$

If $\frac{(n-1) s^{2}}{2 \sigma^{2}}=\mathrm{t}$; then 
International Journal of Soft Computing, Mathematics and Control (IJSCMC), Vol.4, No. 1,February 2015

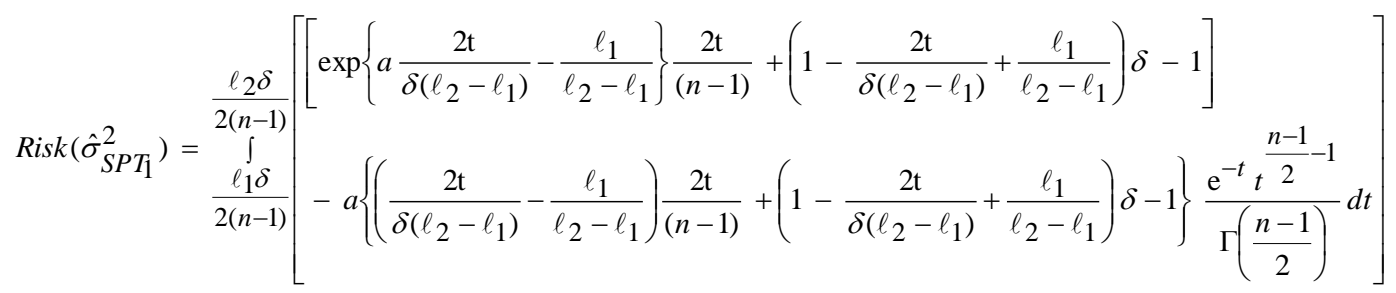

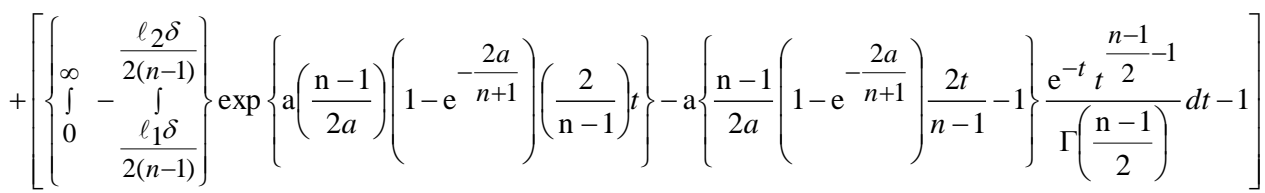

Similarthe expressions for the shrinkage preliminary test estimator $\hat{\sigma}_{S P T_{2}}^{2}$ can be obtained in the future.

The relative efficiency of estimator $\hat{\sigma}_{S P T_{1}}^{2}$ with respect to $\mathrm{P}$ is calculated for $\delta=0.6$ (. 2) (1.2), $\alpha=5 \%, a=.2(.2) 1$ and $n=5(5) 15$ in the table from 3.1 to 3.4. The tablesshow that the estimator $\hat{\sigma}_{S P T_{1}}^{2}$ performs better if $0.6 \leq \delta \leq 1.2, \alpha=5 \%$ and for smaller values of $\mathrm{n}$. The maximum result is at the point $\delta=1$. The AIC information suggest that $\alpha$ should be $16 \%$. The results of $16 \%$ can be calculated, but the above recommendation will give useful results.

The relative efficiency of estimator $\hat{\sigma}_{S P T_{2}}^{2}$ with respect to $\mathrm{P}$ may alsocalculate for $\delta=0.6(.2)$ (1.2), $\alpha=5 \%, \mathrm{a}=.2(.2) 1.0$ and $\mathrm{n}=5(5) 15$.

\section{SHRINKAGEPRELIMINARY TEST ESTIMATOR FOR VARIANCE IN NEGATIVE EXPONENTIAL DISTRIBUTION}

[22] were considered shrinkage estimator for the variance in negative exponential distribution as

$$
T=\mathrm{kx}^{2}+(1-k) \theta_{0}^{2} \quad, 0 \leq \mathrm{k} \leq 1
$$

[21] obtained an improved estimator for $\theta^{2}$ in the class of estimators $Y_{1}=c_{1} \bar{x}^{2}$ under mean square criterion as $Y_{1}^{\prime}=\frac{\mathrm{n}^{2} \bar{x}^{2}}{(n+2)(n+3)}$ with $\operatorname{MSE}\left(Y_{1}^{\prime}\right)=\frac{(4 \mathrm{n}+6) \theta^{4}}{\mathrm{n}(\mathrm{n}+1)}$.

The invariant form of the Linex loss function in the class of the estimator $Y_{1}=c_{1} \bar{x}^{2}$ is

$L\left(a, \Delta^{*}\right)=\mathrm{e}^{\mathrm{a}\left(\frac{\mathrm{c}_{1} \bar{x}^{2}}{\theta^{2}}-1\right)}-\mathrm{a}\left(\frac{\mathrm{c}_{1} \bar{x}^{2}}{\theta^{2}}-1\right)-1$ 
International Journal of Soft Computing, Mathematics and Control (IJSCMC), Vol.4, No. 1,February 2015

Which has the risk $R\left(a, \Delta^{*}\right)=E\left[L\left(a, \Delta^{*}\right)\right]=\mathrm{e}^{-\mathrm{a}} \mathrm{E}\left[\mathrm{e}^{\frac{\mathrm{ac}_{1} \bar{x}^{2}}{\theta^{2}}}\right]-\frac{\mathrm{ac}_{1} n+1}{n}+\mathrm{a}-1$.

$R\left(a, \Delta^{*}\right)=\overline{\mathrm{e}}^{\mathrm{a}} E\left[1+\frac{\mathrm{ac}_{1} \bar{x}^{2}}{\theta^{2}}+\frac{\mathrm{a}^{2} \mathrm{c}_{1}^{2} \bar{x}^{4}}{2 ! \theta^{4}}+\cdots\right]-\frac{\mathrm{ac}_{1}(n+1)}{n}+\mathrm{a}-1$

$R\left(a, \Delta^{*}\right)=\left(1-\mathrm{a}+\frac{\mathrm{a}^{2}}{2 !}-\frac{\mathrm{a}^{3}}{3 !}+\frac{\mathrm{a}^{4}}{4 !}\right)\left[1+\frac{\mathrm{ac}_{1}(n+1)}{n}+\frac{\mathrm{a}^{2} \mathrm{c}_{1}^{2}(n+3)(n+2)(n+1)}{2 ! \mathrm{n}^{3}}+\cdots\right]-\frac{\mathrm{ac}_{1}(n+1)}{n}+\mathrm{a}-1$

If $|a| \rightarrow 0$

$\frac{2}{a^{2}} \mathrm{R}(\mathrm{a}, \Delta)=1-\frac{2 \mathrm{c}_{1}(n+1)}{n}+\frac{\mathrm{c}_{1}^{2}(n+3)(n+2)(n+1)}{\mathrm{n}^{3}}$.

Differentiating with respect to $c_{1}$, thus

$c_{1}=\frac{\mathrm{n}^{2}}{(n+2)(n+3)}$.

For other values of a, value of $c_{1}$ for different values of $\mathrm{n}$ can be obtained by using quadratic equation. Certainty the valuewill be smaller than the minimum value under mean square criterion. Let us suppose that

$c_{1}^{*}=\frac{n^{2} \bar{x}^{2}}{(n+2)(n+3)}\left(1-\frac{\mathrm{c}}{\mathrm{n}}\right)$ Where $\mathrm{c}$ is a constant and less than $\mathrm{n}$.

The propose estimator is

$\hat{\theta}_{S P T}^{2}=\left\{\begin{array}{cc}\mathrm{k} \overline{\mathrm{x}}^{2}+(1-\mathrm{k}) \theta_{0}^{2} & \text { if } \theta=\theta_{0} \text { is accepted } \\ \frac{\mathrm{n}^{2} \overline{\mathrm{x}}^{2}}{(\mathrm{n}+2)(\mathrm{n}+3)}\left(1-\frac{\mathrm{c}}{\mathrm{n}}\right)=\mathrm{Y} & \text { otherwise }\end{array}\right.$

Since $\frac{2 n \bar{x}}{\theta}$ follows the chi - square distribution with $2 \mathrm{n}$ def. The acceptance region can be defined by

$P\left[\ell_{1} \leq \frac{2 n \bar{x}}{\theta_{0}} \leq \ell_{2}\right]=1-\alpha \quad$ and $\quad P\left[\chi_{2 n}^{2} \leq \ell_{1}\right]=P\left[\chi_{2 n}^{2} \geq \ell_{2}\right]=\alpha / 2$

Where the value of $\mathrm{k}$ is 
International Journal of Soft Computing, Mathematics and Control (IJSCMC), Vol.4, No. 1,February 2015

$k=\frac{\frac{2 n \bar{x}}{\theta_{0}}-\ell_{1}}{\ell_{2}-\ell_{1}}$, which shows that $0 \leq \mathrm{k} \leq 1$.

After squaring $\mathrm{k}$ then the shrinkage factor will be smaller than the previous value. Hence $k_{1}=\left(\frac{2 n \bar{x}}{\theta_{0}\left(\ell_{2}-\ell_{1}\right)}-\frac{\ell_{1}}{\ell_{2}-\ell_{1}}\right)^{2}$ can be taken.

One may also consider the square of previous shrinkage factor $\mathrm{k}$ which will be smaller than the previous shrunken factor because $\mathrm{k}$ lies between 0 and 1 .

The proposed shrinkage preliminary test estimators for $\theta^{2}$ in a negative exponential distribution as

$$
\hat{\theta}_{S P T_{1}}^{2}=\left\{\begin{array}{l}
\left(\frac{2 \mathrm{n} \overline{\mathrm{x}}}{\left(\ell_{2}-\ell_{1}\right) \theta_{0}}-\frac{\ell_{1}}{\ell_{2}-\ell_{1}}\right) \overline{\mathrm{x}}^{2}+\left\{1-\left(\frac{2 \mathrm{n} \overline{\mathrm{x}}}{\left(\ell_{2}-\ell_{1}\right) \theta_{0}}-\frac{\ell_{1}}{\ell_{2}-\ell_{1}}\right)\right\} \theta_{0}^{2} \\
\frac{n^{2}(1-c / n) \bar{x}^{2}}{(n+2)(n+3)}=Y
\end{array} \quad \text { if } \frac{\ell_{1} \theta_{0}}{2 n} \leq \overline{\mathrm{x}} \leq \frac{\ell_{2} \theta_{0}}{2 n}\right.
$$

And

$$
\begin{aligned}
& \hat{\theta}_{S P T_{2}}^{2}=\left[\begin{array}{l}
\left(\frac{2 \mathrm{n} \overline{\mathrm{x}}}{\left(\ell_{2}-\ell_{1}\right) \theta_{0}}-\frac{\ell_{1}}{\ell_{2}-\ell_{1}}\right)^{2} \bar{x}^{2}+\left\{1-\left(\frac{2 \mathrm{n} \overline{\mathrm{x}}}{\left(\ell_{2}-\ell_{1}\right) \theta_{0}}+\frac{\ell_{1}}{\ell_{2}-\ell_{1}}\right)^{2}\right\}^{2} \theta_{0}^{2} \text { if } \frac{\ell_{1} \theta_{0}}{2 n} \leq \bar{x} \leq \frac{\ell_{2} \theta_{0}}{2 n} \\
\frac{n^{2}}{(n+2)(n+3)}\left(1-\frac{\mathrm{c}}{\mathrm{n}}\right) \overline{\mathrm{x}}^{2}=\mathrm{Y} \quad \text { otherwise }
\end{array}\right]
\end{aligned}
$$

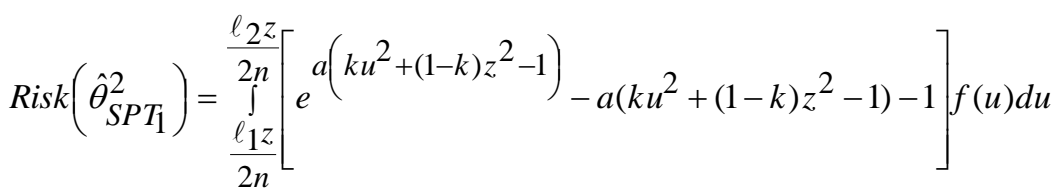

$$
\begin{aligned}
& +\left[\int_{0}^{\frac{\ell_{1} z}{2 n}}+\int_{\frac{\ell_{2} z}{2 n}}^{\infty}\left\{e^{a\left(p u^{2}-1\right)}-a\left(p u^{2}-1\right)-1\right\} f(u) d u\right]
\end{aligned}
$$

The relative efficiency of estimator $\hat{\theta}_{S P T_{1}}^{2}$ with respect to $Y$ is calculated for $\mathrm{z}=0.6(.2)$ (1.2), $\alpha=5 \%, \mathrm{a}=.2, .4, .6,1, \mathrm{n}=3,5,7$ and $\mathrm{c}=1,2$ in the table from 4.1 to 4.8 . The table shows that the 
International Journal of Soft Computing, Mathematics and Control (IJSCMC), Vol.4, No. 1,February 2015

estimator $\hat{\theta}_{S P T_{1}}^{2}$ performs better if $0.6 \leq z \leq 1.2$, for smaller values of a and $\mathrm{n}$ under Linex loss function. The maximum result is at the point $z=1$.

The relative efficiency of estimator $\hat{\theta}_{S P T_{1}}^{2}$ with respect to $\mathrm{Y}$ may also calculate for $\mathrm{z}=0.6(.2)$ (1.2), $\alpha=5 \%, a=.2, .4, .6,1, \mathrm{n}=3,5,7$ and $\mathrm{c}=1,2$.

\section{SCOPE FOR FURTHER RESEARCH}

In this paper author has proposed two estimators for further study. Here author proposed shrinkage preliminary test estimator for the variance in normal distribution and shrinkage preliminary test estimators for the variance $\left(\theta^{2}\right)$ in negative exponential distribution.

\section{REFERENCES}

[1] Adke, S.R. and Gokhale, D.V. (1989) "A note on shrinkage factors in two stage estimation". Comm.Stat.Theo.Meth.18, 633-637.

[2] Adke, S.R., Waikar, V.B.and Schuurman, F.J. (1987) "A two stage shrinkage estimator for the mean of an exponential distribution". Comm. Stat.Theo.Meth.16, 1821-1834.

[3] Arnold, B.C. and AL Bayatti, H.A. (1970) "On double stage estimation of the mean using prior knowledge." Biometrics, 26,787-800.

[4] Bancroft, T.A. (1944) "On biases in estimation due to the use of preliminary test of significance.”Ann.Math.Stat.15, 190-204.

[5] Bhattacharya, S.K and Srivastava, V.K. (1974) "A preliminary test procedure in life testing". Jour. Amer. Stat. Assoc., 69, 726-729.

[6] Ebrahimi, N. and Hosmane, B. (1987) "On shrinkage estimation of the exponential location parameter." Comm. Stat. Theo. Meth.16, 2623-2637.

[7] Gokhale, D.V. (1989) "On two stage shrinkage estimationofthe exponential distribution." Comm. Stat. Theo.Meth.18, 3203-3213.

[8] Goodman, L.A. (1953) "A simple method for improving some estimators."Ann.Math.Stat.24, 114117.

[9] Handa, B.R. and Kambo, N.S. (1990) "A note on modified double stage shrinkage estimator." Comm. Stat. Theo.Meth.19, 4833-4840.

[10] Hirano, K. (1977): Estimation procedures based on preliminary test, shrinkage technique and information criterion. Annals of the Institute of Statistical Mathematics, 29, 21-34.

[11] Hirano, K. (1984) "A preliminary test procedure for the scale parameter of exponential distribution when the selected parameter is unknown. Ann.Inst.Stat.Math.36, 1-9.

[12] Hogg, R.V. (1974) "Adaptive robust procedures: A partial review and some suggestions for future applications and theory." Jour.Amer.Stat.Assoc.69, 909-923.

[13] Huntsberger, D.V. (1955) "A generalization of a preliminary testing procedure of polling data." Ann.Math.Stat.26, 734-743.

[14] Kambo, N. S., Handa, B. R. and AL-Hemyari, Z. A. (1990): On shrunken estimation for exponential scale parameter. Journal of Statistical Planning and Inference, 24, 87-94.

[15] Kambo, N.S., Handa, B.R. and AL-Hemyari, Z.A. (1992) "On Hunts Berger type shrinkage estimator."Comm.Stat.Theo.Meth.21, 823-841.

[16] Karlin, S. (1958) "Admissibility for estimation with quadratic loss. Ann.Math.Stat.29, 406-436,32.

[17] Katti, S.K. (1962) "Use of some a priori knowledge in the estimation of men from double samples."Biometrics 18,139-147. 
International Journal of Soft Computing, Mathematics and Control (IJSCMC), Vol.4, No. 1,February 2015

[18] Lehmann, E.L. (1951) “A generalized concept of unbiased ness.” Ann.Math.Stat.22, 587-597.

[19] Mehta, J.S. and Srinivasan, R. (1971) "Estimation of the mean by shrinkage to a point." Jour.Amer.Stat.Assoc.66, 86-89.

[20] Pandey, B.N. (1997) "Estimator of the scale parameter of the exponential distribution using Linex loss functions." Comm. Stat.Theo.Meth.26, 2191-2200.

[21] Pandey, B.N.and Singh, B.P. (1977) "On estimation of population variance in normal distribution." Jour. Scientific Research, B.H.U., 27,221-225.

[22] Pandey, B.N.and Singh, K.N. (1978) "A pre-test shrinkage estimator of the mean of a normal population.”Jour.Ind.Soc.Agri.Stat.30, 91-98.

[23] Pandey, B.N. and Srivastava, A.K. (2001) "Estimation of variance using asymmetric loss functions." IAPQR.26 (2), 109-123.

[24] Pandey, B.N.and Srivastava, A. R. (1985) "On shrinkage estimation of theexponential scale parameter.”IEEE.Trans.Reliab.R-32, 224-226.

[25] Scolve, Morris and Radhakrishnan (1972), "Non-Optimality of Preliminary-Test Estimators for the Mean of a Multivariate Normal Distribution." Ann.Math.Stat.Vol-43,No.-5, 1481-1490.

[26] Searls, D.T. (1964) "The utilization of a known coefficient of variation in theestimation procedure.”Jour.Amer.Stat.Assoc.59, 1225-1226.

[27] Thompson, J.R. (1968a) "Some shrinkage techniques for estimating the Mean.”Jour.Amer.Stat.Assoc.63, 113-122.

[28] Thompson, J.R. (1968b) "Accuracy borrowing in the estimation of the mean byshrinkage to an interval.” Jour.Amer.Stat.Assoc.63, 953-963.

[29] Varian, H.R. (1975) "A Bayesian approach to real estate assessment." In studies in Bayesian Econometrics and statistics in honor of L. J. Savage, Eds S.E. Feinberge and A, Zellner, Amsterdam, North Holland, 195-208.

[30] Waiker, V.B. and Katti, S.K. (1971) "On a two stage estimate of the mean.”Jour.Amer.Stat.Assoc.66, 76-81,33.

[31] Zellner, A. (1986) "Bayesian estimation and prediction using asymmetric loss functions." Jour.Amer.Stat.Assoc.81, 446-451.

\section{APPENDICES}

Table- 2.1: Relative Efficiency of estimator $\hat{\sigma}_{P T}^{2}$ w.r.to P when $\delta=0.6$ and $\alpha=5 \%$

\begin{tabular}{|c|c|c|c|c|c|}
\hline $\mathrm{a}$ & .2 & .4 & .6 & .8 & 1.00 \\
\hline 5 & & & & & \\
\hline 10 & .932 & .943 & .954 & .966 & .977 \\
\hline 15 & .689 & .700 & .711 & .732 & .732 \\
\hline
\end{tabular}


International Journal of Soft Computing, Mathematics and Control (IJSCMC), Vol.4, No. 1,February 2015

Table- 2.2: Relative Efficiency of estimator $\hat{\sigma}_{P T}^{2}$ w.r.to P when $\delta=0.8$ and $\alpha=5 \%$

\begin{tabular}{|c|c|c|c|c|c|}
\hline $\begin{array}{c}\mathrm{a} \\
\mathrm{n}\end{array}$ & .2 & .4 & .6 & .8 & 1.00 \\
\hline 5 & 2.765 & 2.779 & 2.794 & 2.810 & 2.829 \\
\hline 10 & 2.150 & 2.158 & 2.166 & 2.174 & 2.182 \\
\hline 15 & 1.771 & 1.779 & 1.786 & 1.794 & 1.801 \\
\hline
\end{tabular}

Table-2.3: Relative Efficiency of estimator $\hat{\sigma}_{P T}^{2}$ w.r.to P when $\delta=1.0$ and $\alpha=5 \%$

\begin{tabular}{|c|c|c|c|c|c|}
\hline $\mathrm{a}$ & .2 & .4 & .6 & .8 & 1.00 \\
\hline $\mathrm{n}$ & & & & & \\
\hline 5 & 3.559 & 4.044 & 4.092 & 4.144 & 4.203 \\
\hline 10 & 3.964 & 3.885 & 3.907 & 3.929 & 3.953 \\
\hline 15 & 3.785 & 3.299 & 3.811 & 3.824 & 3.837 \\
\hline
\end{tabular}

Table-2.4: Relative Efficiency of estimator $\hat{\sigma}_{P T}^{2}$ w.r.to P when $\delta=1.2$ and $\alpha=5 \%$

\begin{tabular}{|c|c|c|c|c|c|}
\hline $\mathrm{a}$ & .2 & .4 & .6 & .8 & 1.00 \\
\hline $\mathrm{n}$ & & & & & \\
\hline 5 & 2.742 & 2.732 & 2.722 & 2.712 & 2.704 \\
\hline 10 & 2.125 & 2.106 & 2.088 & 2.069 & 2.051 \\
\hline 15 & & & & & \\
\hline
\end{tabular}

Table-3.1: Relative Efficiency of estimator $\hat{\theta}_{S P T_{1}}^{2}$ w.r.to $P$ when $\delta=0.6 \& \alpha=5 \%$

\begin{tabular}{|c|c|c|c|c|}
\hline $\begin{array}{c}\mathrm{a} \\
\mathrm{n}\end{array}$ & .2 & .4 & .6 & 1.0 \\
\hline 5 & & & & \\
\hline 10 & 1.691 & 1.694 & 1.697 & 1.701 \\
\hline 15 & 1.393 & 1.396 & 1.398 & 1.399 \\
\hline
\end{tabular}


International Journal of Soft Computing, Mathematics and Control (IJSCMC), Vol.4, No. 1,February 2015

Table-3.2: Relative Efficiency of estimator $\hat{\theta}_{S P T_{1}}^{2}$ w.r.to $P$ when $\delta=0.8 \& \alpha=5 \%$

\begin{tabular}{|c|c|c|c|c|}
\hline $\begin{array}{c}\mathrm{a} \\
\mathrm{n}\end{array}$ & .2 & .4 & .6 & 1.0 \\
\hline 5 & & & & \\
\hline 10 & 2.434 & 2.438 & 2.443 & 2.456 \\
\hline 15 & 2.046 & 2.051 & 2.055 & 2.065 \\
\hline
\end{tabular}

Table-3.3: Relative Efficiency of estimator $\hat{\theta}_{S P T_{1}}^{2}$ w.r.to $P$ when $\delta=1.0 \& \alpha=5 \%$

\begin{tabular}{|c|c|c|c|c|}
\hline & .2 & .4 & .6 & 1.0 \\
\hline 5 & & & & \\
\hline 10 & 3.132 & 3.135 & 3.139 & 3.150 \\
\hline 15 & 2.866 & 2.864 & 2.861 & 2.855 \\
\hline
\end{tabular}

Table-3.4: Relative Efficiency of estimator $\hat{\theta}_{S P T_{1}}^{2}$ w.r.to P when $\delta=1.2 \& \alpha=5 \%$

\begin{tabular}{|c|c|c|c|c|}
\hline $\begin{array}{c}\mathrm{a} \\
\mathrm{n}\end{array}$ & .2 & .4 & .6 & 1.0 \\
\hline 5 & 2.682 & 2.663 & 2.643 & 2.601 \\
\hline 10 & 2.289 & 2.169 & 2.248 & 2.204 \\
\hline 15 & 2.023 & 2.004 & 1.985 & 1.946 \\
\hline
\end{tabular}


International Journal of Soft Computing, Mathematics and Control (IJSCMC), Vol.4, No. 1,February 2015

Table 4.1: Relative Efficiency of Estimator $\hat{\theta}_{S P T_{1}}^{2}$ w.r.to $Y$ when $a=0.2, c=1$ \& $\alpha=5 \%$

\begin{tabular}{|c|c|c|c|c|}
\hline Z & .6 & .8 & 1.0 & 1.2 \\
\hline 3 & 1.564 & 3.068 & 6.037 & 3.148 \\
\hline 5 & 1.322 & 2.371 & 5.171 & 3.418 \\
\hline 7 & 1.194 & 1.965 & 4.353 & 3.489 \\
\hline
\end{tabular}

\begin{tabular}{|c|c|c|c|c|}
\hline \multicolumn{1}{|c|}{.6} & .8 & 1.0 & 1.2 \\
\hline 3 & 1.542 & 2.971 & 5.631 & 2.813 \\
\hline 5 & 1.306 & 2.289 & 4.788 & 3.105 \\
\hline 7 & 1.184 & 1.906 & 4.042 & 3.197 \\
\hline
\end{tabular}

Table-4.3: Relative Efficiency of estimator ${ }^{S P T_{1}}$ w.r.to $Y_{\text {when }} a=0.6, c=1 \& \alpha=5 \%$

\begin{tabular}{|c|c|c|c|c|}
\hline \multicolumn{1}{|c|}{.6} & .8 & 1.0 & 1.2 \\
\hline 3 & & & & \\
\hline 5 & 1.521 & 2.877 & 5.247 & 2.519 \\
\hline 7 & 1.291 & 2.310 & 4.426 & 2.825 \\
\hline & 1.174 & 1.845 & 3.749 & 2.934 \\
\hline
\end{tabular}


International Journal of Soft Computing, Mathematics and Control (IJSCMC), Vol.4, No. 1,February 2015

Table-4.4: Relative Efficiency of estimator $\hat{\theta}_{S P T_{1}}^{2}$ w.r.to $Y_{\text {when }} a=1.0, c=1_{\&} \alpha=5 \%$

\begin{tabular}{|c|c|c|c|c|}
\hline \multicolumn{1}{|c|}{.6} & .8 & 1.0 & 1.2 \\
\hline 3 & & & & \\
\hline 5 & 1.484 & 2.704 & 4.563 & 2.043 \\
\hline 7 & 1.157 & 2.062 & 3.783 & 2.364 \\
\hline
\end{tabular}

Table-4.5: Relative Efficiency of estimator $\hat{\theta}_{S P T_{1}}^{2}$ w.r.to $Y_{\text {when }} a=0.2, c=2 \& \alpha=5 \%$

\begin{tabular}{|c|c|c|c|c|}
\hline $\begin{array}{c}\mathrm{z} \\
\mathrm{n}\end{array}$ & .6 & .8 & 1.0 & 1.2 \\
\hline 3 & 1.534 & 2.905 & 5.973 & 3.792 \\
\hline 5 & 1.331 & 2.461 & 6.377 & 4.602 \\
\hline 7 & 1.203 & 2.052 & 5.462 & 4.839 \\
\hline
\end{tabular}

$\hat{\theta}_{S P T_{1}}^{2}$ w.r.to $Y_{\text {when }} a=0.4, c=2 \& \alpha=5 \%$

\begin{tabular}{|c|c|c|c|c|}
\hline $\mathrm{z}$ & .6 & .8 & 1.0 & 1.2 \\
\hline $\mathrm{n}$ & 1.519 & 2.859 & 5.757 & 3.413 \\
\hline 3 & 1.320 & 2.401 & 6.115 & 4.208 \\
\hline 7 & 1.196 & 2.009 & 5.201 & 4.459 \\
\hline
\end{tabular}


International Journal of Soft Computing, Mathematics and Control (IJSCMC), Vol.4, No. 1,February 2015

Table-4.7: Relative Efficiency of estimator $\hat{\theta}_{S P T_{1}}^{2}$ w.r.to $Y_{\text {when }} a=0.6, c=2 \& \alpha=5 \%$

\begin{tabular}{|c|c|c|c|c|}
\hline $\begin{array}{c}\mathrm{z} \\
\mathrm{z}\end{array}$ & .6 & .8 & 1.0 & 1.2 \\
\hline 3 & 1.506 & 2.809 & 5.553 & 3.755 \\
\hline 5 & 1.310 & 2.361 & 5.867 & 3.854 \\
\hline 7 & 1.188 & 1.968 & 4.951 & 4.114 \\
\hline
\end{tabular}

Table-4.8: Relative Efficiency of estimator ${ }^{2}{ }_{S P T_{1}}$ w.r.to $Y_{\text {when }} a=1.0, c=2 \& \alpha=5 \%$

\begin{tabular}{|c|c|c|c|c|}
\hline \multicolumn{1}{|c|}{.6} & .8 & 1.0 & 1.2 \\
\hline 3 & & & & \\
\hline 5 & 1.487 & 2.741 & 5.205 & 2.517 \\
\hline 7 & 1.294 & 2.279 & 5.418 & 3.258 \\
\hline & 1.176 & 1.895 & 4.488 & 3.528 \\
\hline
\end{tabular}

